\title{
Switched/time-based adaptation for second-order sliding mode control *
}

\author{
Alessandro Pisano ${ }^{\mathrm{a}}$, Mara Tanelli ${ }^{\mathrm{b}}$, Antonella Ferrara $^{\mathrm{c}}$ \\ ${ }^{a}$ Department of Electrical and Electronic Engineering, University of Cagliari, Cagliari, Italy \\ ${ }^{\mathrm{b}}$ Dipartimento di Elettronica, Informazione e Bioingegneria, Politecnico di Milano, Piazza L. da Vinci 32, 20133, Milano, Italy \\ ${ }^{\mathrm{c}}$ Dipartimento di Ingegneria Industriale e dell'Informazione, University of Pavia, Pavia, Italy
}

\begin{abstract}
Controlling highly uncertain nonlinear systems is in general a quite difficult task, for which Sliding Mode (SM) control has proved to be an effective option. This brief proposes a SM control strategy which combines a switched policy with a time-based adaptation of the control gain, thereby allowing to effectively deal with a very conservative prior knowledge of the upper bounds on the uncertainties, that usually leads to a large control authority, and related performance degradation. With the proposed approach, the control effort is adjusted online according to the actual magnitude of the uncertain terms, eliminating the conservatism in the selection of the control gain.
\end{abstract}

Key words: Second order sliding mode control; uncertain systems; nonlinear systems; switched systems; adaptation.

\section{Introduction}

Sliding mode (SM) control has long been recognized as a powerful control method to counteract non-vanishing external disturbances and unmodeled dynamics [19], these uncertainty sources being usually time-dependent, highly unpredictable and with arbitrary monotonicity. Yet, in conventional SM control, design relies on the knowledge of worst-case upper bounds of the uncertain terms, which, in most practical cases, result in being highly conservative, with an associated large control authority that may cause a non-negligible chattering. Several approaches to adapt the control effort in SM control have been proposed in the literature over the last decade, see e.g., [15]), [16], [11], which make the amplitude of the control law track the magnitude of the uncertain terms but add significant complexity to the control scheme, along with the transient issues of traditional adaptive algorithms.

Recently, switched algorithms proved to be an efficient choice to achieve performance enhancement with a limited increase in the controller complexity, see e.g., [13], [8], [18]. With reference to SM control, [18] presented

\footnotetext{
* This paper was not presented at any IFAC meeting. Corresponding author M. Tanelli. Tel. +39-02-23993621. Fax +39-0223993412.

Email addresses: pisano@diee.unica.it (Alessandro Pisano), mara.tanelli@polimi.it (Mara Tanelli), antonella.ferrara@unipv. it (Antonella Ferrara).
}

a switched formulation of second order sliding mode ( $\mathrm{S}$ SOSM) controllers, designing a different SOSM control law for each region of the state space in which specific uncertainty bounds are given. In this work, we aim at presenting an original combination between switched and time-based adaptation which allows us to manage the underlying control problem with unique and enhanced features as compared to the existing literature. Specifically, we only ask for very conservative guesses on the upper bounds on the uncertain terms, as the proposed online adaptation allows us to cope with such conservatism and retune the control gain to track the actual uncertainties. Therefore, we can directly compare with some of the adaptive methods that do not ask for knowledge of initial upper bounds, and offer with respect to such purely adaptive solutions - all the advantages of the switched SM philosophy. At the same time, with respect to fixed-structure SM approaches where the controller parameters cannot vary with time, our solution allows to avoid the performance degradation and excessive control authority that comes from tuning the controller parameters based on poor knowledge on the upper bounds on the uncertainties, yielding unnecessarily high gains. A first proposal of the time-based switched adaptation strategy was presented in [3] and [6]. In the present paper, such a strategy is developed for the Suboptimal SOSM control algorithm, [2], and designed jointly with the switched controller. The resulting switched/adaptive control law is shown to yield finite-time convergence to an invariant set, containing the 
origin, defined on the basis of the time-varying uncertainty. Most interestingly, the size of such an invariant set can be $a$ priori estimated based on the controller parameters and on the disturbance characteristics, so that it can be guarantees to be contained in the innermost region of the state-space partition, so that the evolution of the two variables defining the SOSM dynamics is ultimately uniformly bounded. A preliminary and short version of this work, which did not contain the proofs of the algorithm convergence, was presented in [14].

The paper is organized as follows. Section 2 introduces the considered problem and working assumptions. Section 3 presents the proposed control algorithm and its convergence properties, while its performance are discussed in Section 4.

\section{Problem statement}

We deal with nonlinear, single-input single-output, uncertain $n$-th order system that can be transformed into the so-called perturbed chain of integrators form (see e.g., [9]), which takes the expression

$$
\begin{aligned}
& \dot{x}_{i+1}=x_{i}, \quad i=1, \ldots, n-1 \\
& \dot{x}_{n}=\lambda(x(t))+\rho(t)+d(x(t)) u(t),
\end{aligned}
$$

with $x=\left[x_{1}, \ldots, x_{n}\right]^{T}$ being the system state and $\lambda(x(t))$, $\rho(t)$ and $d(x(t))$ being sufficiently smooth nonlinear uncertain functions. Within a sliding mode control framework, assume to select a sliding manifold

$$
s(x(t))=x_{n}(t)+\sum_{i=1}^{n-1} c_{i} x_{i}(t)=0
$$

with the $c_{i}$ being positive constants that make the characteristic equation $z^{n-1}+\sum_{i=1}^{n-1} c_{i} z^{i-1}=0$ have all roots with negative real part. Then it can be shown that (see [2]) if the sliding manifold can be reached in finite time using a second order SM controller with a discontinuous control signal $\dot{u}(t)$, once on the sliding manifold the system behaves as a reduced-order, asymptotically stable linear system.

Consider now the second order uncertain nonlinear system (often referred to as the "auxiliary" system)

$$
\begin{aligned}
& \dot{z}_{1}=z_{2} \\
& \dot{z}_{2}=f(z(t))+h(t)+g(z(t)) v(t),
\end{aligned}
$$

where $z(t)=\left[z_{1}(t) z_{2}(t)\right]^{T} \in \mathbb{R}^{2}$ is the system state, $z_{1}(t)=s(x(t))$ is the sliding variable, $v(t)=\dot{u}(t)$ is the control signal and $f(z(t))$ and $g(z(t))$ are uncertain, sufficiently smooth functions, satisfying all the conditions ensuring existence and uniqueness of the solution [10], and with $h(t)$ being a time-varying perturbation for which a (possibly very conservative) upper bound $H$ is known, i.e.,

$$
|h(t)|<<H, \quad \forall t \geq 0 .
$$

In the case where $h(t)=0$, and it holds that

$$
0<G_{1} \leq g(z(t)) \leq G_{2}, \quad|f(z(t))| \leq F,
$$

the Suboptimal control law (see e.g., [2])

$$
\begin{aligned}
v(t) & =-\alpha V \operatorname{sign}\left(z_{1}(t)-\beta z_{\text {Max }}\right), \quad \beta=\frac{1}{2} \\
\alpha & = \begin{cases}\alpha^{*} & \text { if }\left[z_{1}(t)-\beta z_{\text {Max }}\right]\left[z_{\text {Max }}-z_{1}(t)\right]>0 \\
1 & \text { else, }\end{cases}
\end{aligned}
$$

where $V$ is the control gain, $\alpha$ is the so-called modulation factor, and $z_{M a x}$ is a piecewise constant function representing the value of the last extremal point of $z_{1}(t)$ (an extremal or singular point of the trajectory is defined, see e.g., [1], [5], as a local minimum, local maximum, or a horizontal flex point) makes the system trajectory converge onto the sliding manifold $z_{1}=z_{2}=0$ in finite time provided that the control parameters $\alpha^{*}$ and $V$ are chosen so as to satisfy

$\alpha^{*} \in(0,1] \cap\left(0, \frac{3 G_{1}}{G_{2}}\right), V>\max \left\{\frac{F}{\alpha^{*} G_{1}}, \frac{4 F}{3 G_{1}-\alpha^{*} G_{2}}\right\}$.

In this work, the class of perturbations that can be dealt with by traditional SM control approaches is enlarged, and the auxiliary system is modified as in (3) allowing the presence of the additional time-varying perturbation $h(t)$, along with its associated, possibly very conservative, upper bound $H$. Note that in principle it would be possible to use the suboptimal algorithm with an oversized control magnitude $V$ obtained according to (7) with the constant $F$ being replaced by $F+H$. This approach, however, would lead to unacceptable chattering. Additionally, as in [18], we are going to consider region-dependent uncertainty bounds for the uncertain function $f(\cdot)$. Consider system (3) under the following assumptions.

(i) State-space partitioning: We assume that the state space $\mathcal{Z}$ of system (3) is partitioned into $k$ regions $\mathcal{R}_{i}, i=1, \ldots, k$ defined as

$$
\mathcal{R}_{i}:=\left\{\left(z_{1}, z_{2}\right):\left|z_{1}\right| \leq \bar{z}_{1, i} \text { and }\left|z_{2}\right| \leq \bar{z}_{2, i}\right\}
$$

with $\bar{z}_{j, i-1}>\bar{z}_{j, i}, j=1,2, i=2, \ldots, k-1$, while the outermost region $\mathcal{R}_{1}$ is defined as

$$
\mathcal{R}_{1}:=\left\{\left(z_{1}, z_{2}\right):\left|z_{1}\right| \geq \bar{z}_{1,1} \text { and }\left|z_{2}\right| \geq \bar{z}_{2,1}\right\} .
$$

Further, define $\mathcal{W}_{i}=\partial \mathcal{R}_{i+1}, i=1, \ldots, k-1$. We introduce the regions $\mathcal{Z}_{1} \equiv \mathcal{R}_{1}, \mathcal{Z}_{i}=\mathcal{R}_{i} \backslash \mathcal{R}_{i+1}, i=2, \ldots, k-1$ and $\mathcal{Z}_{k} \equiv \mathcal{R}_{k}$, which are such that $\cup_{i=1, \ldots, k} \mathcal{Z}_{i}=\mathcal{Z}$, and we assume that in each of them different upper and lower bounds for the uncertainties can be defined, to be specified in the following. Note that only the innermost region $\mathcal{Z}_{k}$ contains the origin. 
(ii) State-dependent uncertainty description: Let us assume that in each region $\mathcal{Z}_{i}, i=1, \ldots, k$ a constant upper bound on the uncertain terms is known, i.e., $\forall i=1, \ldots, k$ one can write

$$
0<\overline{\mathcal{G}}_{1, i} \leq g(z(t)) \leq \overline{\mathcal{G}}_{2, i}, \quad|f(z(t))| \leq \overline{\mathcal{F}}_{i}, \quad z \in \mathcal{Z}_{i}
$$

Such upper bounds can be determined owing on the fact that within each of such regions the state norm of the auxiliary system variables is bounded. In presence of the additional time-varying uncertainty affecting (3) no fine tuning of the amplitude of the control law can be made relying on the SSOSM strategy only, and thus in this work we complement it with a time-based adaptation mechanism, which is introduced in the following section.

Remark 1 The assumption of constant bounds for the uncertain functions entering the auxiliary system can be relaxed by exploiting the results presented in [4], where such bounds were replaced by uncertain functions of the system state with linear growth, proving a semiglobal convergence result for the suboptimal algorithm with constant gain.

\section{SOSM controller with combined switched and time- based adaptation}

The time-based adaptation is designed based on the following rationale: consider the sequence of adjacent time intervals $\mathcal{T}_{j}$ of width $T$ defined as $\mathcal{T}_{j} \equiv[(j-1) T, j T)$, $\mathrm{j}=1,2, \ldots$ and a piece-wise constant gain $V_{t b}(t)$ defined as $V_{t b}(t)=V_{M}^{j}, t \in \mathcal{T}_{j}$. Specifically, the amplitude $V_{M}^{j}$ is varied at the end of each time interval $\mathcal{T}_{j}$ according to

$$
\begin{aligned}
& V_{M}^{1}=0 \\
& V_{M}^{j+1}= \begin{cases}\max \left(V_{M}^{j}-\Lambda_{1} T,-V_{k}\right) & \text { if } N_{s w}^{j}(\sigma) \geq N^{*} \\
\min \left(V_{M}^{j}+\Lambda_{2} T, 0\right) & \text { if } N_{s w}^{j}(\sigma)<N^{*},\end{cases}
\end{aligned}
$$

where $N_{s w}^{j}(\sigma)$ is the number of sign commutations of $\sigma(t)=z_{1}(t)-\beta z_{\text {Max }}$ in the interval $\mathcal{T}_{j}$, and $N^{*}$ is an appropriate integer number.

Roughly speaking, at the end of each time interval $\mathcal{T}_{j}$ the time-based control magnitude $V_{t b}(t)$ is decremented stepwise by $\Lambda_{1} T$ if the frequency of the sign commutations of $\sigma(t)$ is sufficiently high, otherwise it is stepwise incremented by $\Lambda_{2} T$. The adaptation logic also includes lower and upper bounds on the control magnitude $\left(-V_{k}\right.$ and 0 , respectively, the first one being the value of the gain amplitude of the switched adaptation rule into the innermost region $R_{k}$ ).

We are now ready to introduce the proposed adaptive switched/time-based SOSM algorithm (STBA-SOSM)

\section{STBA-SOSM Algorithm}

If

$$
z(t) \in \mathcal{Z}_{i} \backslash\left\{\overline{p_{1, i} p_{2, i}} \cup \overline{p_{5, i} p_{6, i}}\right\}, i=1, \ldots, k,
$$

where (see also [18])

$\overline{p_{1, i} p_{2, i}}=\left\{\left(z_{1}, z_{2}\right) \in \delta \mathcal{Z}_{i}: z_{1} \in\left[-\bar{z}_{1, i}+\frac{z_{2}\left|z_{2}\right|}{2 \widetilde{V}_{i}}, z_{2}=\bar{z}_{2, i}\right\}\right.$
$\overline{p_{5, i} p_{6, i}}=\left\{\left(z_{1}, z_{2}\right) \in \delta \mathcal{Z}_{i}: z_{1} \in\left[\bar{z}_{1, i}-\frac{z_{2}\left|z_{2}\right|}{2 \widetilde{V}_{i}}, z_{2}=-\bar{z}_{2, i}\right\}\right.$,

define the control signal as

$$
\begin{aligned}
v_{i}(t) & =-\alpha_{i}\left[V_{i}+V_{t b}(t)\right] \operatorname{sign}\left(z_{1}(t)-\frac{1}{2} z_{\text {Max }}\right) \\
\alpha_{i} & =\left\{\begin{array}{l}
\alpha_{i}^{*} \text { if } \quad\left[z_{1}(t)-\frac{1}{2} z_{\text {Max }}\right]\left[z_{\text {Max }}-z_{1}(t)\right]>0 \\
1 \text { else, }
\end{array}\right.
\end{aligned}
$$

where $V_{i}$ is the control gain for the $i$ th region, $V_{t b}(t)$ is the time based adaptive gain, adjusted according to (11), $\alpha_{i}$ is the $i$ th modulation factor, and all the other quantities have the usual meaning. The control parameters $\alpha_{i}^{*}$ and $V_{i}$ satisfy

$$
\begin{aligned}
& \alpha_{i}^{*} \in(0,1] \cap\left(0, \frac{3 \overline{\mathcal{G}}_{1, i}}{\overline{\mathcal{G}}_{2, i}}\right) \\
& V_{i}>\max \left\{\frac{\overline{\mathcal{F}}_{i}+H}{\alpha_{i}^{*} \overline{\mathcal{G}}_{1, i}}, \frac{4\left(\overline{\mathcal{F}}_{i}+H\right)}{3 \overline{\mathcal{G}}_{1, i}-\alpha_{i}^{*} \overline{\mathcal{G}}_{2, i}}\right\} \\
& V_{i}>V_{\text {Max }}=\max _{j=2, \ldots, k}\left\{\frac{\overline{\mathcal{F}}_{j}+H}{\alpha_{j}^{*} \overline{\mathcal{G}}_{1, j}}\right\}, \quad i=1,2, \ldots, k,
\end{aligned}
$$

while $\widetilde{V}_{i}$ is defined as

$$
\widetilde{V}_{i}=\alpha_{i}^{*} \overline{\mathcal{G}}_{1, i} V_{\text {Max }}-\left(\overline{\mathcal{F}}_{i}+H\right)>0, \forall i=1, \ldots, k,
$$

with $V_{\text {Max }}$ as in the third of (14). If

$$
z(t) \in\left\{\overline{p_{1, i} p_{2, i}} \cup \overline{p_{5, i} p_{6, i}}\right\}, i=1, \ldots, k,
$$

define the control signal as

$$
\begin{array}{r}
v_{i}(t)=-\alpha_{i}\left[V_{i}+V_{t b}(t)\right] \operatorname{sign}\left(z_{1}(t)-\frac{1}{2} z_{\text {Max }}\right) \\
v_{i-1}(t)=\alpha_{i}\left[V_{i}+V_{t b}(t)\right] \operatorname{sign}\left(z_{1}(t)-\frac{1}{2} z_{\text {Max }}\right),
\end{array}
$$

with $\alpha_{i}$ as in (13)

Note that the STBA-SOSM Algorithm relies on the knowledge of the sliding variable derivative $z_{2}(t)$ for the definition of the state-space regions. Such a quantity will be estimated using the finite time convergent Levant's differentiator [12].

For the convergence proof of the closed-loop system, regulated with the STBA-SOSM control law, the following preliminary result is needed. 
Lemma 3.1 Consider the second order auxiliary system (3), regulated by means of the Suboptimal control law, and let $N_{s w}^{j}$ be the number of zero crossings of $\sigma(t)$ during the time interval $\mathcal{T}_{j}$ of length $T$. If condition

$$
N_{s w}^{j}(\sigma) \geq 3
$$

holds, and, in the same interval $\mathcal{T}_{j},\left|\ddot{z}_{1}(t)\right| \leq a_{1}$ for some $a_{1}>0$, then there exist constants $\rho_{1}$ and $\rho_{1}$, independent of $T$, such that the inequalities

$$
\left|z_{1}(t)\right| \leq \rho_{1} T^{2} \quad\left|z_{2}(t)\right| \leq \rho_{2} T
$$

hold along the whole time interval $\mathcal{T}_{j}$.

PROOF. If the function $\sigma(t)=z_{1}(t)-\beta z_{M a x}$ experiences more than two zero crossings within the interval $\mathcal{T}_{j}$, then, by definition of $z_{\operatorname{Max}}$ (the most recent singular value of $\left.z_{1}(t)\right), z_{2}(t)$ features at least two zero crossings within the same interval. Moreover, the time between the attainment of two adjacent singular values, say $z_{M a x, h}$ and $z_{M a x, h+1}$, is bounded (see [4]) as

$$
c_{1} \sqrt{\left|z_{M a x, h}\right|} \leq t_{M_{j+1}}-t_{M_{j}} \leq c_{2} \sqrt{\left|z_{\text {Max }, h}\right|},
$$

for some constants $c_{1}$ and $c_{2}$. By construction of $\mathcal{T}_{j}$, furthermore, within $\left[t_{M_{j}}, t_{M_{j+1}}\right]$ a zero crossing of the switching function $\sigma(t)$ occurs. Thus, the time interval occurring between three consecutive zero crossings of $\sigma(t)$, denoted as $\mathcal{T}_{3 \sigma}$, will be such that

$c_{1}\left(\sqrt{\left|z_{\text {Max }, h}\right|}+\sqrt{\left|z_{\text {Max }, h+1}\right|}\right) \leq \mathcal{T}_{3 \sigma} \leq 3 c_{2} \sqrt{\left|z_{\text {Max }, h}\right|}$

This means that, over the time interval $\mathcal{T}_{j}$

$$
c_{1}\left(\sqrt{\left|z_{\operatorname{Max}, h}\right|}+\sqrt{\left|z_{\operatorname{Max}, h+1}\right|}\right) \leq T
$$

which, in turns, implies that $\sup _{t \in \mathcal{T}_{j}}\left|z_{\text {Max }}\right| \leq \frac{1}{4 c_{1}^{2}} T^{2}$ over $\mathcal{T}_{j}$. Thus, $\left|z_{1}(t)\right| \leq \sup _{t \in \mathcal{T}_{j}}\left|z_{\text {Max }}\right| \leq \frac{1}{4 c_{1}^{2}} T^{2} \forall t \in \mathcal{T}_{j}$, which proves the left inequality in (19). In view of the fact that $z_{2}(t)$ features at least two zero crossings, and taking into account that - by assumption $-\left|\ddot{z}_{1}(t)\right|<a_{1}$, simple time-integration yields the right inequality in (19).

To analyse the properties of the STBA-SOSM algorithm, let us rewrite the second of (3) in the form

$$
\dot{z}_{2}=g(z(t))[\eta(z(t), t)+v(t)]
$$

where

$$
\eta(z(t), t)=\frac{f(z(t))+h(t)}{g(z(t))} .
$$

We can now state the following result
Theorem 1 Consider system (3), with the state space partitioned as in (8). Assume also that, for each $z \in \mathcal{Z}_{i}, i=$ $1, \ldots, k$, the state dependent uncertainties $g(z(t))$ and $f(z(t))$ satisfy (10), and let the time dependent uncertainty be such that (4) holds. Assume also that $\eta(z, t)$ in (24) has a bounded rate of variation in $R_{k}$. Further, consider that a measurement of $z_{1}(t)$ is given, while the estimate of $z_{2}$, denoted by $\hat{z}_{2}$ is computed as

$$
\begin{aligned}
\dot{\zeta} & =\hat{z}_{2}, \\
\hat{z}_{2} & =-\gamma_{0}\left|\zeta-z_{1}\right|^{1 / 2} \operatorname{sign}\left(\zeta-\mathrm{z}_{1}\right)+\nu_{1} \\
\dot{\nu}_{1} & =-\gamma_{1} \operatorname{sign}\left(\zeta-\mathrm{z}_{1}\right),
\end{aligned}
$$

where $\gamma_{0}$ and $\gamma_{1}$ are positive constants chosen such that

$$
\gamma_{1}>L, \quad \gamma_{0}^{2} \geq 4 L \frac{\gamma_{1}+L}{\gamma_{1}-L}, \quad L \geq \max _{j=1,2, \ldots, k}\left\{\overline{\mathcal{F}}_{j}+\overline{\mathcal{G}}_{2, j} V_{j}\right\} .
$$

Apply now the STBA-SOSM Algorithm with

$$
\Lambda_{1}>0, \quad \Lambda_{2}>\Lambda_{1}+2 P_{k}, \quad N^{*} \geq 3 .
$$

Then, for a sufficiently small $T$ and for some positive constants $b_{1}, b_{2}$, after a finite-time transient the following inequalities hold

$$
\left|z_{1}(t)\right| \leq b_{1} T^{2}, \quad\left|\dot{z}_{2}(t)\right| \leq b_{2} T
$$

PROOF. The tuning formulas (26) represent the well known tuning inequalities of the Levant's differentiator [12], in which $L$ represents an upper bound to the Lipschitz constant of $z_{2}$. First, notice that the STBA-SOSM control law in Algorithm 3 guarantees that

$$
|v(t)| \leq \max _{i=1,2, \ldots, k}\left|\alpha_{i}\left[V_{i}+V_{t b}(t)\right]\right|
$$

Further, note that, by definition, $\alpha_{i} \in(0,1]$ and $V_{t b}(t) \in$ $\left[-V_{k}, 0\right]$ due to the adopted upper and lower bounds in (11). Thus, the relationship (29) can be further manipulated as

$$
|v(t)| \leq \max _{i=1,2, \ldots, k} V_{i}
$$

According to the second of (3), and taking into account the state uncertainty description (10) and the upper bound on the uncertainty $h(t)$, it readily follows that $\left|\dot{z}_{2}\right| \leq \max _{j=1,2, \ldots, k}\left\{\overline{\mathcal{F}}_{j}+\overline{\mathcal{G}}_{2, j} V_{j}\right\} \equiv L$, which shows that the parameter $L$ in (26) is indeed a Lipschitz constant of $z_{2}$. After a transient of finite duration, which can be made arbitrarily small by choosing a value of $L$ in (26) which is large enough, the estimated and actual state $z_{2}$ will be coincident. However, unless the initial conditions of the system trajectories already belong to a close vicinity of the origin, during the transient phase there will be no frequent 
sign commutations of $\sigma(t)$. Hence, the time-based magnitude $V_{t b}(t)$ will be kept constant and equal to its initial zero value until a vicinity of the origin, the size of which depends on the parameter $T$, is reached. With a sufficiently small $T$, this neighborhood can be forced to be entirely contained in the innermost $\mathcal{Z}_{k}=\mathcal{R}_{k}$. Outside region $\mathcal{R}_{k}$, therefore, the controller implements the purely switched S-SOSM adaptation method, investigated in [18], which guarantees that the region $\mathcal{R}_{k}$ is finite time attractive and positively invariant. Once within the innermost region $\mathcal{R}_{k}$, the actual system trajectory in the $z_{1} O z_{2}$ plane will approach the origin, as guaranteed by the fact that the region $\mathcal{R}_{k}$ is invariant and that the switched control gain $V_{k}$ dominates the actual uncertainties. During this convergence process, the frequency of the sign commutations of $\sigma(t)$ will correspondingly and progressively increase, according to (21) and in view of the contraction condition $\left|z_{\operatorname{Max}, h+1}\right| \leq \gamma\left|z_{\operatorname{Max}, h}\right|, \gamma<1$, enforced by the Suboptimal algorithm. Ideally, the frequency of the sign commutations of $\sigma(t)$ tends to infinity as the closed-loop trajectory approaches the origin. Therefore, condition (18) is kept at some finite $j=M_{1}$, and the stepwise reduction of $V_{M}^{j}$ is activated starting from the end of the time interval $\mathcal{T}_{M_{1}}$. Define now

$$
N_{k}=\sup _{t \geq 0, z(t) \in \mathcal{R}_{k}} \eta(z(t), t)=\frac{\overline{\mathcal{F}}_{k}+H}{\overline{\mathcal{G}}_{1, k}}
$$

and note that, with the state evolution being restricted to the innermost region $\mathcal{R}_{k}$, the dominance condition of the Suboptimal algorithm control gain is expressed as

$$
V_{M}^{j}+V_{k} \geq \Gamma N_{k}, \quad \Gamma=\max \left\{\frac{1}{\alpha^{*}}, \frac{4 \mathcal{G}_{1, k}}{3 \mathcal{G}_{1, k}-\mathcal{G}_{2, k}}\right\},
$$

where the left hand side of (32) includes both the switched and the time-based components. By relying on the fact that $N_{s w}^{M_{1}}(\sigma) \geq N^{*}$, at the end of the time interval $\mathcal{T}_{M_{1}}$ (i.e., at the time instant $\left.t=M_{1} T\right)$ the actual value of the time-based adaptive control magnitude $v_{t b}\left(M_{1} T\right)=V_{M}^{M_{1}}$, summed with the value of the switched control gain $V_{k}$ will be "dominating" the actual upper bound of $\eta(z(t), t)$ in accordance with (32), as

$$
V_{M}^{M_{1}}+V_{k} \geq \Gamma\left|\eta\left(z\left(M_{1} T\right), M_{1} T\right)\right| .
$$

The dominance over the uncertainties (formalized by condition (32)) will be lost after a finite number of intervals, and at some $j=M_{2}>M_{1}$ the 2-sliding criterion (18) will be violated. This implies that, at the end of the preceding time interval $\mathcal{T}_{M_{2}-1}$, a dominance inequality analogous to (33) holds, i.e.,

$$
V_{M}^{M_{1}}+V_{k} \geq \Gamma\left|\eta\left(z\left(\left(M_{2}-1\right) T\right),\left(M_{2}-1\right) T\right)\right| .
$$

In view of Lemma 3.1, at the end of the time interval $\mathcal{T}_{M_{2}-1}$ the variables $z_{1}$ and $z_{2}$ are bounded as in (19) with

$$
\rho_{1}=\sup _{t \in \mathcal{T}_{M_{2}-1}}\left|\dot{z}_{2}(t)\right|=\overline{\mathcal{G}}_{2, k}\left[N_{k}+V_{M}^{M_{2}-1}+V_{k}\right] .
$$

At the end of $\mathcal{T}_{M_{2}+1}$, i.e., one time interval after the violation of the 2-sliding criterion (18), the magnitude of the uncertainty $\eta(z(t), t)$ will be such that

$$
\begin{array}{r}
\left|\eta\left(z\left(\left(M_{2}+1\right) T\right),\left(M_{2}+1\right) T\right)\right| \leq \\
\left|\eta\left(z\left(\left(M_{2}-1\right) T\right),\left(M_{2}-1\right) T\right)\right|+2 P_{k} T
\end{array}
$$

which considers the bound on the rate of variation of $\eta(z, t)$ in (24). On the other hand, the adaptive gain magnitude will be increased at the end of the interval $\mathcal{T}_{i, M_{2}}-1$, and decreased at the end of the successive one $\mathcal{T}_{i, M_{2}+1}$, which means that

$$
V_{M}^{M_{2}+1}>V_{M}^{M_{2}-1}+T\left(\Lambda_{2}-\Lambda_{1}\right) .
$$

Therefore, considering (34) and (37), and provided that $\Lambda_{2}$ is such that

$$
\Lambda_{2}>\Lambda_{1}+2 P_{k},
$$

the dominance condition (32) will be already restored at the end of the time interval $\mathcal{T}_{i, M_{2}+1}$, i.e., one time interval after the violation of the 2-sliding criterion (18) occurred. While $V_{M}^{j}$ continues to grow, waiting for the 2-sliding criterion (18) to be restored, which will happen after a finite number of time intervals, the contractive rotations of the system trajectories in the $z_{1}-z_{2}$ plane can be evaluated by studying the limit trajectories induced by the Suboptimal controller starting from the initial condition (19). Lengthy - yet straightforward - algebraic computations show that the transient deviations of $z_{1}$ and $z_{2}$ fulfil inequalities of the type (28), where constants $b_{1}$ and $b_{2}$ depend on the uncertainty bounds and on the controller parameters, but do not depend on $T$. The process of losing and successively restoring the dominance over the uncertainties will continue iteratively in the subsequent time intervals, preserving inequalities (28). As a final step, one must choose $T$ such that the invariant region described by the inequalities (28) is contained within the innermost region $R_{k}$. To achieve this, $T$ has to be chosen sufficiently small, and in accordance with

$$
T \leq \min \left\{\sqrt{\frac{\bar{z}_{1, k}}{b_{1}}}, \frac{\bar{z}_{2, k}}{b_{2}}\right\}
$$

Remark 2 Some "practically oriented" guidelines for finding an effective tuning of the $N^{*}$ parameter are given. The interval length $T$ has to be chosen first, according to the desired accuracy degree. Then, an experimental test using the fixed-gain version of the controller and inspecting the actual "average" number of switches of the quantity $\sigma(t)$ along the time windows of length $T$ has to be done. This observed average value has then to be reduced (e.g., dividing it by two ) to derive $N^{*}$. The rationale for this procedure is that the average switching frequency of $\sigma(t)$ will decrease when the magnitude of the discontinuous controller gain will be diminished. 


\section{Simulation results}

To illustrate the performance of the proposed STBA-SOSM algorithm, consider the dynamical model of the single-link manipulator with flexible joint and negligible damping [17], i.e.,

$$
\begin{aligned}
& I \ddot{q}_{1}(t)+M g L \sin \left(q_{1}(t)\right)+k\left(q_{1}(t)-q_{2}(t)\right)=0 \\
& J \ddot{q}_{2}(t)-k\left(q_{1}(t)-q_{2}(t)\right)=u(t)+w(t)
\end{aligned}
$$

where $q_{1}(t)$ and $q_{2}(t)$ are the angular positions of the link and motor, respectively, $I$ and $J$ are moments of inertia, $k$ is the stiffness constant, $M$ is the total mass, $L$ is a distance, $u(t)$ is the input torque, while $w(t)$ is a bounded unmeasurable disturbance. The control objective is to steer the angular positions and velocities of the manipulator to zero while avoiding excessive transient oscillations of the link in spite of the presence of the disturbance term. System (40) can be rewritten as a fourth-order nonlinear model, which can be transformed into the Brunowsky normal form through a global diffeomorphism (see [10]) yielding

$$
\left\{\begin{aligned}
\dot{p}_{i}(t) & =p_{i+1}(t), \quad i=1,2,3 \\
\dot{p}_{4}(t) & =-\left(\frac{M g L}{I} \cos \left(p_{1}(t)\right)+\frac{k}{I}+\frac{k}{J}\right) p_{3}(t) \\
& +\frac{M g L}{I}\left(p_{2}^{2}(t)-\frac{k}{J}\right) \sin \left(p_{1}(t)\right)+\frac{k}{I J}(u(t)+w(t))
\end{aligned}\right.
$$

To perform the simulation tests, the same parameters used in [7] have been adopted, that is, $M g L=1, I=0.08$, $J=0.005, k=0.3$. Note that the adopted stiffness value corresponds to the case of a very flexible joint, which makes the regulation task quite hard. The disturbance input is taken as $w(t)=5 \sin (2 t)$. The chosen sliding manifold is $z_{1}(t)=$ $p_{4}(t)+15 p_{3}(t)+75 p_{2}(t)+125 p_{1}(t)=0$, which assigns an asymptotically stable behavior to the associated reducedorder zero dynamics, with three poles all located at -5 . From the definition of the sliding manifold, an auxiliary system of the form (3), i.e.,

$$
\begin{aligned}
& \dot{z}_{1}=z_{2} \\
& \dot{z}_{2}=f(p(t), \dot{p}(t))+\frac{k}{I J}(v(t)+\dot{w}(t)),
\end{aligned}
$$

where function $f(\cdot, \cdot)$, which is supposed to be uncertain, can be straightforwardly obtained from (40), and the auxiliary control variable $v(t)$ denotes the time derivative of the actual input torque, i.e., $v(t)=\dot{u}(t)$. Although $\dot{w}(t)$ is uniformly bounded, in magnitude, by constant $H=10$, it is assumed that the much more conservative upper bound $H=50$ is available $a$ priori to the designer. The initial conditions are $x(0)=(0.5,-0.5,0.5-0.5)$. The drift term function $f(\cdot)$ can be bounded by a constant term if one assumes that the state variables remain bounded, which appears reasonable considering the physical meaning of such variables in the considered robotic system. Four regions $\mathcal{R}_{1}$, $\mathcal{R}_{2}, \mathcal{R}_{3}, \mathcal{R}_{4}$ are considered, with parameters $\bar{z}_{1,1}=10$, $\bar{z}_{1,2}=6, \bar{z}_{1,3}=2, \bar{z}_{2,1}=450, \bar{z}_{2,2}=350, \bar{z}_{2,2}=150$. All simulations have been done using the Euler integration method with a fixed integration step of $10^{-4} s$. The joint velocities $\dot{q}_{1}$ and $\dot{q}_{2}$ have been estimated on-line via real-time differentiation of the position signals $q_{1}$ and $q_{2}$ using Levant's differentiators [12]. In TEST 1, the purely switched S-SOSM controller, without time-based adaptation has been implemented. The parameters of the switched adaptation are: $V_{1}=100, V_{2}=90, V_{3}=80, V_{4}=60, \alpha_{i}=0.9, \forall i$. The parameters of estimator (25) are taken as $\gamma_{1}=120$, $\gamma_{0}=70$. The left plot in Figure 1 shows the closed-loop trajectory in the $z_{1}-z_{2}$ plane. It can be seen that the initial conditions lie in the region $\mathcal{R}_{1}$, and that the inner regions $\mathcal{R}_{2}, \mathcal{R}_{3}$ and $\mathcal{R}_{4}$ are progressively entered until the invariant innermost region $R_{4}$ is reached. The left plot of Figure 1 shows a zoomed view of the trajectory. The large values taken by $z_{1}(t)$ and $z_{2}(t)$ during the transient are due to the high gains used in the sliding variable definition, selected to have a fast convergence to the reduced order sliding mode dynamics. Figure 2-left depicts the time-varying magnitude of the switched control gain, showing its progressive decrease as the inner regions are entered. The switched gain quickly decreases during the initial transient and eventually settles to the value $V_{4}$ once the trajectory enters the innermost invariant region $R_{4}$.
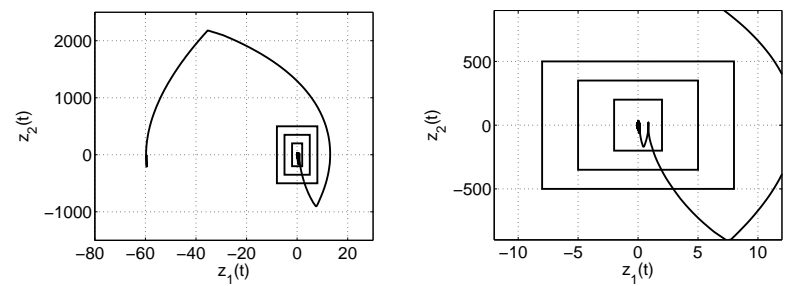

Fig. 1. Trajectory in the $z_{1}-z_{2}$ plane in TEST 1 . Left plot: overall trajectory. Right plot: zoomed trajectory.
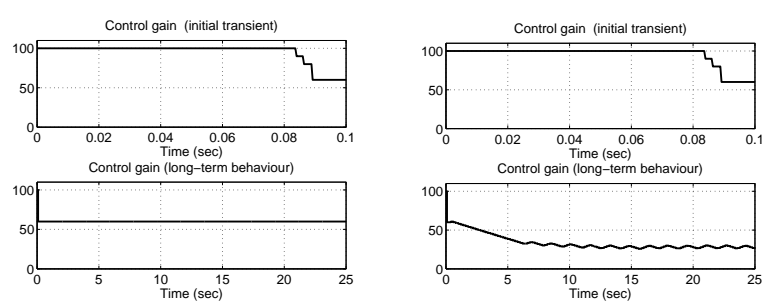

Fig. 2. Time history of the control gain in TEST 1 (left plots) and TEST 2 (right plots). Upper plots: initial transient. Lower plots: long-term evolution.

In TEST 2, the proposed STBA-SOSM algorithm has been implemented. The parameters of the switched adaptation are the same as in TEST 1, while those of the time-based component of the algorithm are $\Lambda_{1}=5, \Lambda_{2}=7, N^{*}=10, T=$ $50 \mathrm{~ms}$. The resulting trajectory in the $z_{1}-z_{2}$ plane is indistinguishable from that achieved in TEST 1, and omitted for brevity. Figure 2-right depicts the overall magnitude of the control gain (including both the switched and the timebased component). The upper plot shows that the transient behaviour is the same as that obtained in the TEST 1, while 
the lower plot shows that as soon as the sliding mode behaviour is established in a vicinity of the second order sliding set $z_{1}(t)=z_{2}(t)=0$ the control gain further reduces and, at steady state, it successively increases and decreases, tracking the fluctuations of the actual uncertainties. The plots in Figure 3 show the converging behaviour of $z_{1}(t)$ and $q_{1}(t)$, which are both quickly steered to zero. It is worth to underline the prompt and oscillation-free regulation of the arm coordinate $q_{1}(t)$, shown in the right plot, which is achieved in spite of the high flexibility of the joint and in presence of oscillatory disturbances too. Figure 4 highlights the further benefit of using the combined switched and time based adaptation that the time evolution of the control input $u(t)$ is less corrupted by chattering.

In TEST 3, the STBA-SOSM algorithm has been implemented using a reduced value, $T=25 \mathrm{~ms}$, of the temporal adaptation window, half of that used in TEST 2. The aim of this test is to verify the relation (28). As clear from Fig. 5 , the accuracy of $z_{1}(t)$ is four times higher in the TEST 3 as compared to that achieved in the TEST 2, which confirms the quadratic dependence of the $z_{1}$ accuracy on the $T$ parameter.
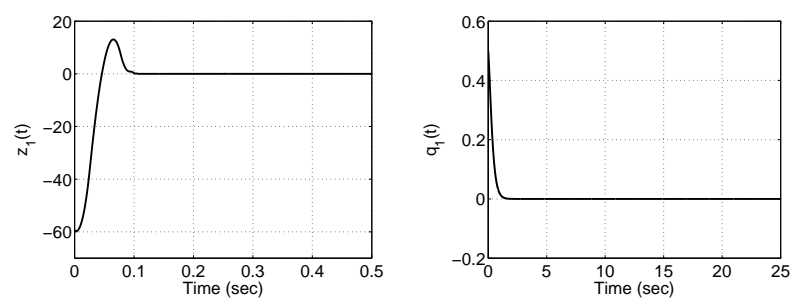

Fig. 3. Transient behaviour of $z_{1}(t)$ (left plot) and $q_{1}(t)$ (right plot) in TEST 2.

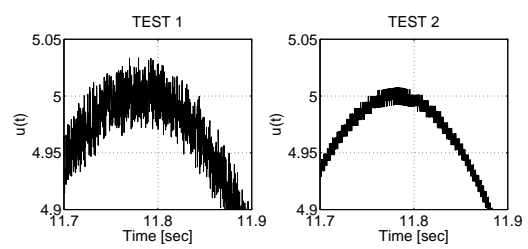

Fig. 4. Zoom of $u(t)$ in TEST 1 (left plot) and TEST 2 (left plot).

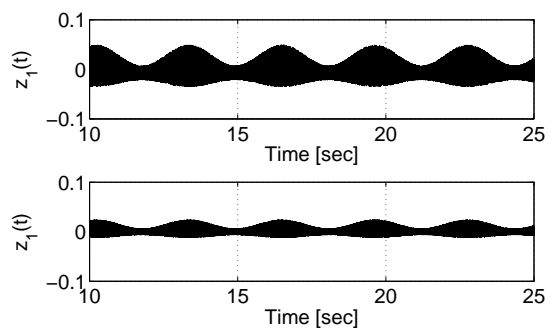

Fig. 5. Upper plot: zoom of $z_{1}(t)$ in TEST 2 with $\mathrm{T}=0.05 \mathrm{~s}$. Lower plot: zoom of $z_{1}(t)$ in TEST 3 with $\mathrm{T}=0.025 \mathrm{~s}$.

\section{Concluding remarks}

In this paper, a combined switched/time-based adaptation strategy for a SOSM controller has been presented, which allows dealing with both state-dependent and time-varying uncertainties for which only (possibly very conservative) upper bounds are known. The proposed algorithms features the desired convergence properties, as the closed-loop trajectory reaches in finite-time an invariant set containing the origin, the size of which can be explicitly linked to the controller parameters.

\section{References}

[1] G. Bartolini, A. Ferrara, and E. Usai. On output tracking control of uncertain non linear second order systems. Automatica, 33(12):22032212, 1997.

[2] G. Bartolini, A. Ferrara, and E. Usai. Chattering avoidance by second-order sliding mode control. IEEE Transactions on Automatic Control, 43(2):241-246, 1998.

[3] G. Bartolini, A. Levant, A. Pisano, and E. Usai. 2-sliding mode with adaptation. In Proceeding of the 1999 IEEE Mediterranean Conference on Control and Systems, Haifa, Israel, 1999.

[4] G. Bartolini, A. Pisano, A. Ferrara, and E. Usai. On the convergence properties of a 2-sliding control algorithm for nonlinear uncertain systems. International Journal of Control, 74(7):718-731, 2001.

[5] I. Boiko, L. Fridman, A. Pisano, and E. Usai. Analysis of chattering in systems with second-order sliding modes. IEEE Transactions on Automatic Control, 52(11):2085-2102, 2007.

[6] L. Capisani, A. Ferrara, and A. Pisano. Second-order sliding mode control with adaptive control authority for the tracking control of robotic manipulators. In Proceeding of the 2011 IFAC World Congress, Milan, Italy, 2011.

[7] M. Corless and S. Zenieh. A new control design methodology for robotic manipulators with flexible joints. In Proceedings of the American Control Conference, volume 6, pages 4316-4320, 1995.

[8] M.L. Corradini and G. Orlando. Transient Improvement of Variable Structure Controlled Systems Via Multi-Model Switching Control. Journal of Dynamic Systems, Measurement, and Control, 124(2):321326, 2002.

[9] F. Dinuzzo and A. Ferrara. Higher Order Sliding Mode Controllers With Optimal Reaching. IEEE Transactions on Automatic Control, 54(9):2126-2136, 2009.

[10] H.K. Khalil. Nonlinear Systems. 2nd Edition, Prentice Hall, Upper Saddle River, New Jersey, 1996.

[11] J. Kochalummoottil, Y.B. Shtessel, J.A. Moreno, and L. Fridman. Output feedback adaptive twisting control: A Lyapunov design. In Proceedings of the American Control Conference, pages 6172-6177, Montreal, Canada, 2012.

[12] A. Levant. Robust exact differentiation via sliding mode technique. Automatica, 34(3):379-384, 1998.

[13] L. Magni, R. Scattolini, and M. Tanelli. Switched model predictive control for performance enhancement. International Journal of Control, 81(12):1859-1869, 2008.

[14] A. Pisano, M. Tanelli, and A. Ferrara. Combined switched/time-based adaptation in second order sliding mode control. In Proceedings of the 52nd IEEE Conference on Decision and Control, pages 4272 4277, Firenze, Italy, 2013.

[15] F. Plestan, Y. Shtessel, V. Br égeault, and A. Poznyak. New methodologies for adaptive sliding mode control. International Journal of Control, 83(9):1907-1919, 2010. 
[16] Y.B. Shtessel, J.A. Moreno, F. Plestan, L.M. Fridman, and A.S. Poznyak. Super-twisting adaptive sliding mode control: A Lyapunov design. In Proceedings of the 49th IEEE Conference on Decision and Control, pages 5109-5113, Atlanta, GA, 2010.

[17] M. W. Spong and M. Vidyasagar. Robot dynamics and control. John Wiley \& Sons, 2008.

[18] M. Tanelli and A. Ferrara. Enhancing robustness and performance via switched second order sliding mode control. IEEE Transactions on Automatic Control, 58(4):962-974, 2013.

[19] V. Utkin, J. Guldner, and J. Shi. Sliding-Mode Control in Electromechanical Systems. Taylor \& Francis, London, U.K., 1999. 